PHYSICAL REVIEW B 92, 119904(E) (2015)

\title{
Erratum: Chiral gauge field and axial anomaly in a Weyl semimetal [Phys. Rev. B 87, 235306 (2013)]
}

Chao-Xing Liu, Peng Ye, and Xiao-Liang Qi

(Received 28 August 2015; published 18 September 2015)

DOI: 10.1103/PhysRevB.92.119904

PACS number(s): 71.90.+q, 03.65.Vf, 73.43.-f, 75.50.Pp, 99.10.Cd

A small revision for the anomaly equation $\partial_{\mu} j^{\mu L(R)}=(-) \frac{1}{32 \pi^{2}} \epsilon^{\lambda \rho \mu \nu} f_{\lambda \rho}^{L(R)} f_{\mu \nu}^{L(R)}$, as well as the definition of the field strength $f_{\mu \nu}^{L(R)}$, in the paragraph just above Eqs. (2) and (3) is required. The anomaly equation should be $\partial_{\mu} j^{\mu L(R)}=$ $-(+) \frac{1}{32 \pi^{2}} \epsilon^{\lambda \rho \mu \nu} f_{\lambda \rho}^{L(R)} f_{\mu \nu}^{L(R)}$ for left-handed (right-handed) fermions and the corresponding field strength for left-handed and right-handed gauge field should be defined as $f_{\mu \nu}^{L(R)}=\partial_{\mu}\left(A_{\nu}+a_{\nu L(R)}\right)-\partial_{\nu}\left(A_{\mu}+a_{\mu L(R)}\right)$. Our main results, i.e., Eqs. (2) and (3) as well as other parts of the paper, are valid. 